\title{
Reconstrucción lingual con colgajo libre radial: A propósito de un caso
}

\section{Lingual reconstruction with radial forearm flap: About a case}

\author{
Francisca Fernández A. ${ }^{1,2}$, Daniela Vicencio S. ${ }^{1}$, Jaime Bermeo S. ${ }^{1}$, Ciro Bustos . $^{3}$
}

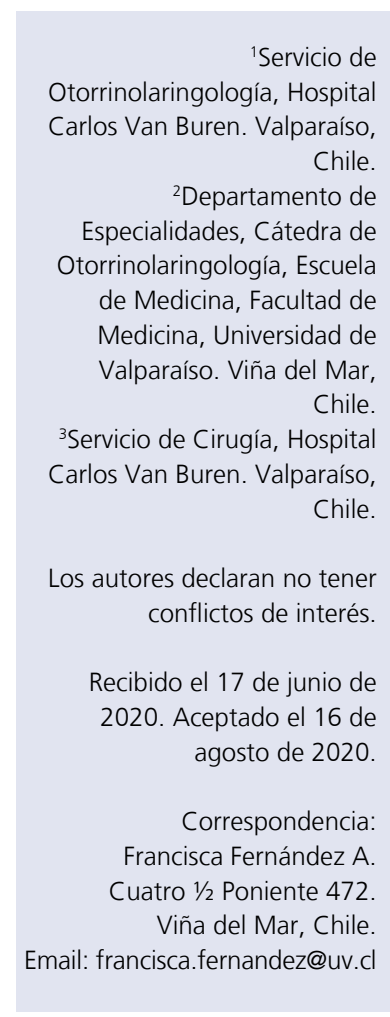

\section{Resumen}

El cáncer de lengua es una patología con una importante morbilidad e impacto en la calidad de vida del paciente, afectando la masticación, deglución, comunicación y gusto, por lo que requiere de un equipo multidisciplinario para establecer la rehabilitación adecuada. Se necesita realizar resecciones amplias para asegurar márgenes negativos, y si el cirujano considera que existe un volumen remanente insuficiente para obliterar la cavidad oral, la realización de un colgajo será necesario para mantener la función deglutoria. Se presenta el caso de un paciente de 76 años, masculino, con antecedentes de tabaquismo suspendido hace 23 años, que presenta una lesión ulcerada dolorosa en el borde derecho de la lengua móvil, la biopsia informa un carcinoma epidermoide, etapificado T2N0M0, por lo que se decide realizar hemiglosectomía derecha más disección cervical y reconstrucción con colgajo libre radial.

Palabras clave: Cáncer de lengua, glosectomía, colgajo libre radial.

\begin{abstract}
Tongue cancer is a pathology with significant morbidity and impact on the quality of life of the patients. It affects chewing, swallowing, communication, and taste. Therefore, a multidisciplinary team is required to establish adequate rehabilitation. Extensive resections are needed to ensure negative margins. If the surgeon considers that there is insufficient remaining volume to obliterate the oral cavity, flap surgery will be carried out to maintain the swallowing function. We present a case report of a 76-year-old male patient with a history of smoking cessation of 23 years. He presents a painful ulcerated lesion on the right edge of the mobile tongue. The biopsy reports an epidermoid carcinoma, staged T2NOMO which led to perform a right hemiglosectomy, cervical dissection, and reconstruction with free radial forearm flap.

Keywords: Tongue cancer, glosectomy, free radial forearm flap.
\end{abstract}

\section{Introducción}

El cáncer de lengua corresponde al sitio más frecuente de presentación dentro del cáncer de cavidad oral y representa el 1\% de todos los cánceres nuevos diagnosticados en Estados Unidos, con una tasa de mortalidad de 0,7 por cada 100.000 personas según los datos aportados por la National Cancer Institute ${ }^{1}$. Se estima que durante el año 2020 existirán 17.660 nuevos casos, observándose un incremento anual de 2,1\% en los últimos 10 años en esta población ${ }^{1}$. En Chile, los registros sobre la incidencia o prevalencia a nivel nacional son escasos, describiéndose una incidencia estimada de 3,2 por 100.000 hombres y 1,2 casos por 100.000 mujeres para el conjunto de pacientes con cáncer de cavidad oral y orofaringe. Por otro lado, la lengua sería el sitio más frecuente en este grupo de pacientes ${ }^{2}$.

El cáncer de lengua predomina en hombres, con un pico de presentación entre los 55 y 64 años ${ }^{1}$. Destacan como factores de riesgo el consumo de tabaco y alcohol, incrementando el riesgo hasta en un 50\% en comparación a la población que no consume ${ }^{3}$. Otro factor de riesgo eventualmente a considerar es el virus papiloma humano (VPH). En un estudio de 
Gillison y cols. del año 2000 se encontró genoma viral en células tumorales, pero aún no existe consenso con respecto al rol del VPH en el carcinoma espinocelular de cavidad oral ${ }^{4}$.

Usualmente los pacientes consultan por una masa exofítica, ulcerada o indurada de la lengua, siendo lo más relevante, la evaluación de la profundidad de esta lesión. La profundidad de la invasión (DOI) se ha agregado en la última edición del sistema de estapificación AJCC $^{5}$, luego que numerosos estudios demostraran una relación significativa entre el DOI y el riesgo de metástasis ganglionar ${ }^{6-8}$.

El tratamiento de elección es la resección quirúrgica con márgenes amplios y eventual reconstrucción ${ }^{9}$, siendo este último uno de los problemas más complejos de resolver en la cirugía de cabeza y cuello ${ }^{10}$. Su participación durante la deglución, fonación, articulación y gusto, hacen que una adecuada reconstrucción sea fundamental para la calidad de vida de los pacientes sometidos a resecciones por estos tumores ${ }^{11}$. Cuando el cirujano establezca que existe un volumen remanente insuficiente para obliterar la cavidad oral, un colgajo será necesario para mantener la función deglutoria ${ }^{12}$.

\section{Caso Clínico}

Paciente de 67 años de sexo masculino, con antecedentes de hipertensión arterial en tratamiento y tabaquismo suspendido hace 23 años, con una frecuencia de una cajetilla/ día por 32 años. Fue derivado desde Atención Primaria al Servicio de Cirugía Maxilofacial por un cuadro de 3 meses de una lesión dolorosa en borde lateral derecho de lengua móvil. La biopsia incisional informa carcinoma epidermoide, derivándose al Servicio de Otorrinolaringología para manejo. Al examen físico destaca desdentado total superior y parcial inferior; en tercio medio y posterior del borde lateral derecho de lengua móvil, presenta lesión ulcerada de bordes indurados, de $3 \mathrm{~cm}$ anteroposterior y $2 \mathrm{~cm}$ céfalo-caudal, que a la palpación es dolorosa e impresiona infiltrar aproximadamente $1 \mathrm{~cm}$ en profundidad, sin sobrepasar la línea media, y sin comprometer el piso de la boca. Movilidad lingual conservada, pero dolorosa. No se palpan adenopatías cer- vicales. Se realiza tomografía computada (TC) de cuello y tórax donde se describe un tumor en borde lateral derecho de lengua móvil, de 2 por $1 \mathrm{~cm}$. No se observan metástasis cervicales o a distancia. A la resonancia magnética (RM) cervical se observa lesión de 3,5,2 y $1 \mathrm{~cm}$ en su eje anteroposterior, vertical y transverso respectivamente.

Se presenta a comité oncológico con el diagnóstico de carcinoma epidermoide moderadamente diferenciado de cavidad oral en el borde lateral derecho de lengua móvil, T2N0M0, decidiéndose hemiglosectomía parcial derecha con reconstrucción lingual con colgajo libre microvascularizado radial, disección cervical supraomohioidea bilateral y traqueostomía. La biopsia posoperatoria informa, glosectomía parcial de $6,3 \mathrm{~cm}$ en su diámetro anteroposterior, con una tumoración nodular focalmente indurada que mide $5 \mathrm{~cm}$ anteroposterior por $3 \mathrm{~cm}$ de diámetro vertical, con una profundidad de $2,6 \mathrm{~cm}$, correspondiente a un carcinoma epidermoide moderadamente diferenciado. Las ampliaciones de todos sus bordes se encontraban libres de tumor, al igual que los ganglios cervicales. Con estos antecedentes, se reetapifica como T3N0M0 y se decide realizar radioterapia posoperatoria. El paciente evolucionó de forma favorable, sin morbilidad en el sitio donante y con una inteligibilidad y deglución satisfactoria. Sin embargo, refiere ausencia de sensibilidad en la zona reconstruida.

\section{Discusión}

Existen diferentes abordajes para la reconstrucción de los defectos por hemiglosectomías. Para tomar la decisión más adecuada se debe considerar el tamaño del tejido, la composición y grosor de éste ${ }^{13}$. Los objetivos de la reconstrucción incluyen: obliteración de la cavidad oral, entendiéndose esto como el contacto que deben hacer las diferentes superficies mucosas cuando la boca está cerrada, contacto de la lengua reconstruida con el paladar y la premaxila para la adecuada articulación de sonidos; movilización de la saliva y recuperación de la sensibilidad, movilidad, forma y volumen lingual ${ }^{10}$. Para lograr esto es fundamental seguir los siguien- 
tes principios: sobrecorregir el volumen del defecto, reconstrucción del piso de boca con tejidos delgados y lingual con tejidos gruesos y restauración del volumen sin bloquear los distintos surcos 9 .

Dependiendo del grado de resección, se pueden recomendar distintos procedimientos para la reconstrucción: cierre primario, cierre por segunda intención, colgajos cervicales (submentonianos y platisma), colgajos pediculados regionales (como miocutáneo pectoral y fasciocutáneo del deltopectoral) y colgajos libres (fasciocutáneo radial, ulnar, anterolateral del muslo, entre otros) ${ }^{14}$ (Tabla 1).

El colgajo fasciocutáneo del antebrazo es considerado la primera opción para la reconstrucción ${ }^{15}$. Fue realizado por primera vez hace 41 años para una cobertura de mano, y desde ahí su uso se ha extendido utilizando colgajos libres en tronco, extremidades inferiores y reconstrucción en cabeza y cuello, esófago y pene. Puede cubrir lesiones complejas dadas su capacidad de incluir distintos tejidos como piel, hueso, tendones, músculos y nervios para reparar defectos, ya sea con flujo directo, retrógrado o libre ${ }^{16}$.

La reconstrucción en tumores avanzados de lengua móvil mediante el uso de colgajos libres, permite realizar resecciones más amplias que aseguren márgenes negativos. Se promueve el uso de colgajo libre radial por ser un colgajo maleable, flexible y resistente en el tiempo y que se puede utilizar independiente de la edad ${ }^{9}$. El componente cutáneo es ideal para la cobertura de mucosas al iniciar un proceso de metaplasia. Además, se ha demostrado mejores resultados estéticos y funcionales, incluyendo la mejoría en la deglución y sensibilidad, en comparación a colgajos regionales ${ }^{16}$.

En cuanto a la técnica quirúrgica, es primordial identificar la arteria radial, la cual corre bajo los músculos braquiorradial y flexor radial del carpo, lateral al septum intermuscular. Desde su origen emite perforantes que irrigarán músculos, huesos $\mathrm{y} 3 / 4$ partes de la piel del antebrazo. La técnica quirúrgica consiste en

Tabla 1. Recomendación de colgajo a utilizar según tipo de glosectomía

Tipo 1.

Hemiglosectomía subtotal: Resección

de hasta un tercio

del volume total de la lengua
Tipo 2. Hemiglosectomía: Mitad de la lengua y puede comprometer su base
Tipo 3. Hemiglosectomía extendida: Incluye parte del piso anterior de la boca contralateral, y/o pilar amigdalino y/o rafe lingual
Tipo 4. Glosectomía subtotal: Todo el piso de la bboca, los dos tercios anteriores de la lengua y puede incluir la mitad de la base de la lengua
Tipo 5. Glosectomía total: Totalidad de la lengua y el piso de la boca
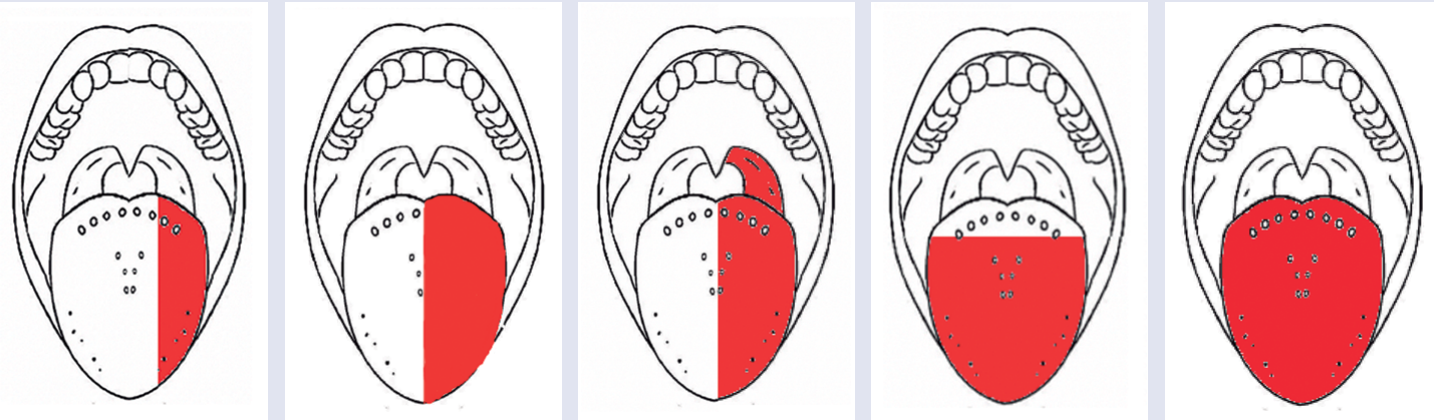

Colgajos locales. Ejemplo: Mesolabial o el mucoso-labial
Colgajos cutáneos o fasciocutáneos libres microvasculares, como el radial y el lateral de brazo
Se prefieren colgajos bultosos como: colgajo musculocutáneo de recto abdominal y fasciocutáneo anterolateral de muslo 
diseñar un colgajo según los requerimientos del defecto (el tamaño máximo es de 20 x 10 $\mathrm{cm}$ como zona dadora), realizar una incisión en la piel y tejido celular subcutáneo, teniendo precaución de no lesionar los vasos perforantes, posteriormente se debe identificar la arteria radial, venas acompañantes y nervio radial; finalmente, se debe individualizar y seccionar el pedículo con isla cutánea ${ }^{16}$.

Las complicaciones quirúrgicas inmediatas pueden incluir infección, sangrado, aspiración, ruptura de la herida, pérdida del colgajo, fístula y necrosis del remanente de lengua posresección. En cuanto a las complicaciones a largo plazo se describen alteraciones de deglución y habla, que dependen de qué tan óptima sea la reconstrucción ${ }^{9}$. La zona dadora también puede sufrir complicaciones como dehiscencia de herida, exposición de tendón, defectos estéticos y la necesidad de curaciones prolongadas $^{16}$.

\section{Conclusión}

La reconstrucción ha cambiado drásticamente en los últimos treinta años desde que se realizaron los primeros colgajos libres para reconstruir defectos en cabeza y cuello. En ese sentido, la colaboración interdisciplinaria y organización de equipos dedicados a la cirugía de cabeza y cuello es fundamental. Muchas veces los equipos quirúrgicos se pueden ver tentados en comprometer los márgenes oncológicos durante la resección en orden de preservar suficiente tejido y no reconstruir. Este tipo de prácticas se deben considerar impresentables el día de hoy. De esta manera es fundamental, realizar una evaluación preoperatoria cuidadosa con TC, RM y reconstrucciones en $3 \mathrm{D}$, lo cual nos permitirá evaluar el tamaño y profundidad del defecto; realización de múltiples biopsias intraoperatorias para no comprometer los márgenes; y un equipo quirúrgico capaz de realizar la reconstrucción en un mismo tiempo, con el objeto de que los defectos no presenten fibrosis secundaria y distorsión. Finalmente, se recomienda realizar colgajos libres por sobre los colgajos regionales, puesto que presentarían mejores resultados estéticos $y$ funcionales, favoreciendo una mayor inteligibilidad y función deglutoria.

\section{Bibliografía}

1. Howlader N, Noone AM, Krapcho M, et al. SEER Cancer Statistics Review, 1975-2017, National Cancer Institute. Bethesda, MD, Disponible en: https://seer. cancer.gov/csr/1975_2017. Consultado el 13 de julio de 2020.

2. Santelices Ch MJ, Cárcamo IM, Brenner AC, Montes FR. Cáncer oral en Chile: Revisión de la literatura. Rev Med Chile 2016;144(6):758-766. doi:10.4067/ S0034-98872016000600011

3. García A, Domínguez A, García J, Cancela G, Torres J, Esparza G. Revisión y puesta al día en cáncer de lengua. Av Odontoestomatol. 2013;29(5):255-269. https://doi.org/10.4321/S021312852013000500005.

4. Gillison ML, Koch WM, Capone RB, et al. Evidence for a causal association between human papillomavirus and a subset of head and neck cancers. J Natl Cancer Inst. 2000;92(9):709-720. doi: 10.1093/ jnci/92.9.709.

5. Lydiatt WM, Patel SG, O'Sullivan B, et al. Head and Neck cancers-major changes in the American Joint Committee on cancer eighth edition cancer staging manual. CA Cancer J Clin. 2017;67(2):122-137. doi: $10.3322 /$ caac. 21389 .

6. Mair MD, Shetty R, Nair D, et al. Depth of invasion, size and number of metastatic nodes predicts extracapsular spread in early oral cancers with occult metastases. Oral Oncol. 2018;81:95-99. doi: 10.1016/j. oraloncology.2018.04.015.

7. Mitani S, Tomioka T, Hayashi R, Ugumori T, Hato N, Fujii S. Anatomic Invasive Depth Predicts Delayed Cervical Lymph Node Metastasis of Tongue Squamous Cell Carcinoma. Am J Surg Pathol. 2016;40(7):934-942. doi:10.1097/ PAS.0000000000000667.

8. Tam S, Amit M, Zafereo M, Bell D, Weber RS. Depth of invasion as a predictor of nodal disease and survival in patients with oral tongue squamous cell carcinoma. Head Neck. 2019;41(1):177-184. doi: 10.1002/hed.25506.

9. Nieto L, Pedraza C. Reconstrucción Microquirúrgica de la lengua. Rev Chil Cir. 2016;68(2):180-185. doi: 10.4067/S0718-40262016000200013.

10. Hanasono MM, Matros E, Disa JJ. Important aspects of head and neck reconstruction. Plast Reconstr Surg. 2014;134(6):968e-980e. doi: 10.1097/ PRS.0000000000000722.

11. Lu CC, Tsou YA, Hua CH, Tsai MH. Free flap reconstruction for early stage tongue squamous cell carcinoma: surgical margin and recurrence. Acta Otolaryngol. 2018;138(10):945-950. doi: 10.1080/00016489.2018.1490030.

12. Chepeha DB, Teknos TN, Shargorodsky J, et al. Rectangle tongue template for reconstruction of the hemiglossectomy defect. Arch Otolaryngol Head 
Neck Surg. 2008;134(9):993-998. doi: 10.1001/ archotol.134.9.993.

13. Chepeha DB, Spector ME, Chinn SB, et al. Hemiglossectomy tongue reconstruction: Modeling of elevation, protrusion, and functional outcome using receiver operator characteristic curve. Head Neck. 2016;38(7):1066-1073. doi: 10.1002/ hed.24417.

14. Skoner J, Horning J, Day T. Reconstruction of Partial Glossectomy Defects. En: T. Day and D. Girod, ed., Oral Cavity Reconstruction, 1st ed.
New York: Taylor \& Francis Group. 2006, pp. 205222.

15. Biglioli F, Liviero F, Frigerio A, Rezzonico A, Brusati R. Function of the sensate free forearm flap after partial glossectomy. J Craniomaxillofac Surg. 2006;34(6):332-339. doi: 10.1016/j.jcms.2006.04.005.

16. Andrades P, Calderón M, Danilla S, Benítez S, Erazo C, Sepúlveda S. Colgajo radial: experiencia del equipo de Cirugía Plástica de la Universidad de Chile. Rev Chil Cir. 2011; 63(5):459-467. doi: 10.4067/S071840262011000500004. 